\title{
MULTISCALE MODELLING AND COMPUTATION OF MICROSTRUCTURES IN MULTI-WELL PROBLEMS
}

\author{
ZHIPING LI
}

\author{
LMAM \& SCHOOL OF MATHEMATICAL SCIENCES, \\ PEKING UNIVERSITY, BEIJING 100871, P.R.CHINA
}

\begin{abstract}
A multiscale model and numerical method for computing microstructures with large and inhomogeneous deformation is established, in which the microscopic and macroscopic information is recovered by coupling the finite order rank-one convex envelope and the finite element method. The method is capable of computing microstructures which are locally finite order laminates. Numerical experiments on a double well problem show that plenty of stress free large deformations can be achieved by microstructures consisting of piecewise simple twin laminates.
\end{abstract}

\section{INTRODUCTION}

It is well known that, below the transformation temperature, many elastic crystals transform to a lower symmetric martensitic phase which allows them to have stress free large deformations exhibiting microstructures consisting of fine mixtures of martensitic variants. For the static problem of martensitic microstructures, the well known geometrically nonlinear continuum theory $[1,2]$ leads to the consideration of minimizing the elastic energy

$$
F(u ; \Omega)=\int_{\Omega} f(\nabla u(x)) d x
$$

in a set of admissible deformations

$$
\mathbb{U}\left(u_{0} ; \Omega\right)=\left\{u \in W^{1, p}\left(\Omega ; R^{m}\right): u=u_{0}, \text { on } \partial \Omega_{0}\right\},
$$

1991 Mathematics Subject Classification. 73C50,65K10,65N30.

Key words and phrases. multiscale modelling, multi-well problem, energy minimization, laminated microstructure, stress free large deformation.

The research was supported in part by the Special Funds for Major State Basic Research Projects (G1999032804) and RFDP of China. 
where $\Omega \subset R^{n}$, with $n=2$ or 3 , is a bounded open set with a Lipschitz continuous boundary $\partial \Omega, \partial \Omega_{0}$ is a subset of $\partial \Omega$ with positive $(n-1)$-dimensional measure and $n<p<\infty$, and where the Ericksen-James elastic energy density $f(\cdot)$ has several symmetry related energy wells $[1,2,5]$ and is of the form [4]

$$
f(\nabla u)=\Phi(C)
$$

where $C=(\nabla u)^{T} \nabla u \in \mathbb{S}^{n}=\left\{A \in R^{n \times n}: A^{T}=A\right\}$, which is the set of symmetric matrices in $R^{n}$, is the right Cauchy-Green strain tensor.

For properly given linear boundary data $u_{0}$, the minimizing sequences of the elastic energy $F(\cdot ; \Omega)$ in $\mathbb{U}\left(u_{0} ; \Omega\right)$ will essentially consist of finely laminated twins which are in different energy wells $[1,2]$. The numerical computation of laminated microstructures is by no means trivial $[16,23]$ and has been attracting many researches in the past two decades (see [23] for a survey on the classical conforming and nonconforming finite element methods, see [9, 10, 11, 12, 17, 18, 19, 20, 25] among many others for other approaches).

For large nonlinear macroscopic deformations consisting of inhomogeneous microstructure, the numerical computation encounters much greater challenge. In [15], an attempt was made by coupling a coarse mesh approximation of macroscopic deformation with a fine mesh approximation of microstructure. However, since the scale of microstructure is much smaller than that of macroscopic deformation, a high accuracy approximation with such a method usually leads to unbearable computational work. In the present paper, considering the multiscale nature of the deformation, we establish a multiscale computational model which uses the mesh transformation method [20, 21, 22] for the approximation of the macroscopic deformation and the finite order rank-one convex envelope scheme [18] to reveal the information of the microstructure. The idea of applying the mesh transformation method is to involve the mesh distribution into the minimization procedure to limit the mesh dependence of the finite element approximation, which can often be a serious problem in numerical computation of microstructures $[6,7,17,23]$. Theoretically, the multiscale method given in this paper is capable of computing microstructures which are locally finite order laminates as observed in many elastic crystals. 
The rest of the paper is organized as follows. In section 2, the finite order rank-one convex envelopes are defined and analyzed, and the corresponding relaxed problem is proposed. In section 3, the multiscale computational model and the numerical algorithm are established and analyzed. In section 4, numerical experiments on a double well problem are given to show that plenty of stress free large deformations can be achieved by microstructures consisting of piecewise simple twin laminates, which justifies the necessity of developing such kind of multiscale methods as established in this paper.

\section{Relaxation by finite order RANK-ONe CONVEX ENVElopes}

As is well known that one of the main difficulties in the numerical approximation of the non-convex variational problem is that the infimum of $F(\cdot ; \Omega)$ in $\mathbb{U}\left(u_{0} ; \Omega\right)$ is generally unattainable and the minimizing sequences produce finer and finer oscillations which leads to the so called gradient Young measure solutions $[1,2,23]$. To avoid this difficulty, one may consider the problem of minimizing the relaxed energy functional

$$
Q F(u ; \Omega)=\int_{\Omega} Q f(\nabla u(x)) d x
$$

in $\mathbb{U}\left(u_{0} ; \Omega\right)$, where $Q f(\cdot)$ is the quasiconvex envelope of $f(\cdot)$, i.e. the greatest quasiconvex function less than or equal to $f[8,24]$. Under certain general growth and coerciveness conditions, the relaxed problem is solvable and the solutions, termed as relaxed minimizers, turn out to be the weak limits of minimizing sequences of the original problem $[1,2,24]$, which can also be viewed as the macroscopic version of the gradient Young measure solutions. Other than the loss of microscopic information, the fatal shortcoming of this approach in applications is that quasiconvex envelope $Q f(\cdot)$ is generally unavailable and its numerical computation is no less difficult than the original problem.

Notice that typical microstructures observed in martensite crystals are laminated microstructures, which include simple laminates and some finite orders of laminates in laminates. Since these laminated microstructures can be completely resolved by the finite order rank-one convex envelopes $R_{k} f(\cdot)$ given below (see also $[18]$ ), it is well founded that, in the computation of martensitic microstructures, 
we consider the problem of minimizing the relaxed energy functional

$$
R_{k} F(u ; \Omega)=\int_{\Omega} R_{k} f(\nabla u(x)) d x
$$

in $\mathbb{U}\left(u_{0} ; \Omega\right)$.

Let $f: R^{m n} \rightarrow R^{1} \cup\{\infty\}$ be continuous.

Definition 2.1. Let $R_{1} f: R^{m n} \rightarrow R^{1} \cup\{\infty\}$ be defined by

$$
\begin{array}{r}
R_{1} f(A)=\inf \left\{\lambda f\left(A_{0}\right)+(1-\lambda) f\left(A_{1}\right): \lambda \geq 0, A=\lambda A_{0}+(1-\lambda) A_{1},\right. \\
\left.\operatorname{rank}\left(A_{0}-A_{1}\right) \leq 1\right\},
\end{array}
$$

and let $R_{k} f: R^{m n} \rightarrow R^{1} \cup\{\infty\}$ be defined by

$$
R_{k} f(A)=R_{1}\left(R_{k-1} f\right)(A) .
$$

$R_{k} f$ is called the $k$-th order rank-one convex envelope of $f$.

Definition 2.2. The sequence

$$
\left(\lambda_{i_{1}}, \lambda_{i_{1} i_{2}}, \ldots, \lambda_{i_{1} i_{2} \cdots i_{k}}, A_{i_{1} i_{2} \cdots i_{k}}\right), \quad i_{\nu} \in\{0,1\}, \quad 1 \leq \nu \leq k
$$

is said to satisfy $\left(\mathrm{R}_{k}\right)$ if

$$
\begin{aligned}
& \lambda_{i_{1} \cdots i_{\nu}} \geq 0, \quad \forall \nu=1, \cdots, k, \quad \lambda_{0}+\lambda_{1}=1, \\
& \lambda_{i_{1} \cdots i_{\nu-1} 0}+\lambda_{i_{1} \cdots i_{\nu-1} 1}=1, \quad \text { for } \nu=2, \cdots, k,
\end{aligned}
$$

and if the following conditions are satisfied:

(i): $A_{i_{1} i_{2} \cdots i_{k}} \in R^{m n}, \operatorname{rank}\left(A_{i_{1} \cdots i_{k-1} 0}-A_{i_{1} \cdots i_{k-1} 1}\right) \leq 1$,

(ii): let $A_{i_{1} \cdots i_{\nu}}=\lambda_{i_{1} \cdots i_{\nu} 0} A_{i_{1} \cdots i_{\nu} 0}+\lambda_{i_{1} \cdots i_{\nu} 1} A_{i_{1} \cdots i_{\nu} 1}$, then

$$
\operatorname{rank}\left(A_{i_{1} \cdots i_{\nu-1} 0}-A_{i_{1} \cdots i_{\nu-1} 1}\right) \leq 1, \forall \nu=k-1, \cdots, 2 .
$$

For direct computation of the $k$-th order rank-one convex envelopes, the following equivalent definition can be used.

Definition 2.3. $R_{k} f: R^{m n} \rightarrow R^{1} \cup\{\infty\}$ is said to be the $k$-th order rank-one convex envelope of $f$, if

$$
R_{k} f(A)=\inf \left\{\sum_{i_{1}, \ldots, i_{k}=0}^{1} \lambda_{i_{1}} \lambda_{i_{1} i_{2}} \cdots \lambda_{i_{1} i_{2} \cdots i_{k}} f\left(A_{i_{1} i_{2} \cdots i_{k}}\right):\right.
$$




$$
\begin{aligned}
& \sum_{i_{1}, \ldots, i_{k}=0}^{1} \lambda_{i_{1}} \lambda_{i_{1} i_{2}} \cdots \lambda_{i_{1} i_{2} \cdots i_{k}} A_{i_{1} i_{2} \cdots i_{k}}=A \\
& \left.\quad\left(\lambda_{i_{1}}, \lambda_{i_{1} i_{2}}, \ldots, \lambda_{i_{1} i_{2} \cdots i_{k}}, A_{i_{1} i_{2} \cdots i_{k}}\right) \text { satisfy }\left(\mathrm{R}_{k}\right)\right\} .
\end{aligned}
$$

Lemma 2.1. [18] We have

$$
f \geq R_{1} f \geq R_{2} f \geq \cdots \geq R_{k} f \geq \cdots \geq R f \geq Q f,
$$

where $R f$ is called the rank-one convex envelope of $f[8]$, and

$$
\lim _{k \rightarrow \infty} R_{k} f(A)=R f(A), \forall A \in R^{m n} .
$$

Theorem 2.1. [18] For any $k \geq 1$ and any $u_{0} \in W^{1, p}\left(\Omega ; R^{m}\right)$, we have

$$
\inf _{u \in \mathbb{U}\left(u_{0}, \Omega\right)} \int_{\Omega} R_{k} f(\nabla u(x)) d x=\inf _{u \in \mathbb{U}\left(u_{0}, \Omega\right)} \int_{\Omega} f(\nabla u(x)) d x .
$$

One of the advantages of relaxation by the finite order rank-one convex envelopes is that $R_{k} f(\cdot)$ is computable. Let $A \in R^{m n}$, define

$$
A_{i_{1} \cdots i_{k}}=A+\sum_{j \in I_{i_{1} \cdots i_{k}}} \gamma_{j^{-}}\left((1-i(j)) \sin ^{2}\left(\tau_{j^{-}}\right)-i(j) \cos ^{2}\left(\tau_{j^{-}}\right)\right) \phi_{j^{-}} \otimes \theta_{j^{-}},
$$

where the index set $I_{i_{1} \cdots i_{k}}=\left\{i_{1}, i_{1} i_{2}, \ldots, i_{1} i_{2} \cdots i_{k}\right\}$ and

$$
\begin{gathered}
i(j)=i_{\nu}, \quad \text { if } j=i_{1} \cdots i_{\nu} \quad \text { for } \nu=1,2, \cdots k, \\
j^{-}= \begin{cases}-, & \text {if } j=i_{1}, \\
i_{1} \cdots i_{\nu-1}, & \text { if } j=i_{1} \cdots i_{\nu},\end{cases} \\
\theta_{j^{-}} \in S^{n-1}, \quad \phi_{j^{-}} \in S^{m-1}, \quad \tau_{j^{-}} \in S^{1} \text { and } \gamma_{j^{-}} \in R^{1},
\end{gathered}
$$

and define

$$
\lambda_{j}=(1-i(j)) \cos ^{2}\left(\tau_{j^{-}}\right)-i(j) \sin ^{2}\left(\tau_{j^{-}}\right), \quad j \in I_{i_{1} \cdots i_{k}},
$$

then, it is easily verified that $\left(\lambda_{i_{1}}, \lambda_{i_{1} i_{2}}, \ldots, \lambda_{i_{1} i_{2} \cdots i_{k}}, A_{i_{1} \cdots i_{k}}\right)$ satisfy $\left(\mathrm{R}_{k}\right)$ and

$$
A=\sum_{i_{1}, \ldots, i_{k}=0}^{1} \lambda_{i_{1}} \lambda_{i_{1} i_{2}} \cdots \lambda_{i_{1} i_{2} \cdots i_{k}} A_{i_{1} i_{2} \cdots i_{k}} .
$$


Theorem 2.2. [18] Let $A \in R^{m n}$. Let $A_{i_{1} \cdots i_{k}}$ and $\lambda_{j}, j \in I_{i_{1} \cdots i_{k}}$ be defined by (2.9) and (2.13) respectively. Then, we have

$$
R_{k} f(A)=\inf \sum_{i_{1}, \ldots, i_{k}=0}^{1} \lambda_{i_{1}} \lambda_{i_{1} i_{2}} \cdots \lambda_{i_{1} \cdots i_{k}} f\left(A_{i_{1} \cdots i_{k}}\right),
$$

where the infimum is taken among all $\left\{\left(\theta_{j^{-}}, \phi_{j^{-}}, \tau_{j^{-}}, \gamma_{j^{-}}\right), j \in I_{i_{1} \cdots i_{k}}\right\}$ given by (2.12).

By Theorem 2.2, to calculate $R_{k} f(A)$ is to solve a nonlinear unconstrained optimization problem with $(m+n)\left(2^{k}-1\right)$ variables. For example, the first and second order rank-one convex envelope can be written as

$$
\begin{aligned}
& R_{1} f(A)=\inf \left\{\cos ^{2}(\tau) f\left(A+\gamma \sin ^{2}(\tau) \phi \otimes \theta\right)+\sin ^{2}(\tau) f\left(A-\gamma \cos ^{2}(\tau) \phi \otimes \theta\right)\right\}, \\
& R_{2} f(A)=\inf \left\{\cos ^{2}\left(\tau_{0}\right) \cos ^{2}(\tau) f\left(A+\gamma \sin ^{2}(\tau) \phi \otimes \theta+\gamma_{0} \sin ^{2}\left(\tau_{0}\right) \phi_{0} \otimes \theta_{0}\right)\right. \\
&+\sin ^{2}\left(\tau_{0}\right) \cos ^{2}(\tau) f\left(A+\gamma \sin ^{2}(\tau) \phi \otimes \theta-\gamma_{0} \cos ^{2}\left(\tau_{0}\right) \phi_{0} \otimes \theta_{0}\right) \\
&+\cos ^{2}\left(\tau_{1}\right) \sin ^{2}(\tau) f\left(A-\gamma \cos ^{2}(\tau) \phi \otimes \theta+\gamma_{1} \sin ^{2}\left(\tau_{1}\right) \phi_{1} \otimes \theta_{1}\right) \\
&+\left.\sin ^{2}\left(\tau_{1}\right) \sin ^{2}(\tau) f\left(A-\gamma \cos ^{2}(\tau) \phi \otimes \theta-\gamma_{1} \cos ^{2}\left(\tau_{1}\right) \phi_{1} \otimes \theta_{1}\right)\right\} .
\end{aligned}
$$

Denote

$$
\Delta_{k}=\left\{\left(\theta_{j^{-}}, \phi_{j^{-}}, \tau_{j^{-}}, \gamma_{j^{-}}\right) \in S^{n-1} \times S^{m-1} \times S^{1} \times R^{1}, j \in I_{i_{1} \cdots i_{k}}\right\},
$$

let $\delta_{k} \in \Delta_{k}$ and

$$
f_{k}\left(A ; \delta_{k}\right)=\sum_{i_{1}, \ldots, i_{k}=0}^{1} \lambda_{i_{1}} \lambda_{i_{1} i_{2}} \cdots \lambda_{i_{1} \cdots i_{k}} f\left(A_{i_{1} \cdots i_{k}}\right),
$$

where $A_{i_{1} \cdots i_{k}}$ and $\lambda_{j}, j \in I_{i_{1} \cdots i_{k}}$ are defined by (2.9) and (2.13), then the $k$-th order rank-one convex envelope can be written as

$$
R_{k} f(A)=\inf _{\delta_{k} \in \Delta_{k}} f_{k}\left(A, \delta_{k}\right) .
$$

Another key advantage of relaxation by the finite order rank-one convex envelopes is that the quantities involved in the optimization have obvious physical meanings. For example, $\theta$ s are the unit normals to the interfaces between the laminates, and $\cos ^{2}(\tau) \mathrm{s}$ and $\sin ^{2}(\tau) \mathrm{s}$ are the volume fractions of the corresponding 
laminates, etc. In fact, the information on microstructures consisting of laminates in laminates of order no greater than $k$ can be recovered by computing $R_{k} f(A)$.

Theorem 2.3. Let $f: R^{m n} \rightarrow R^{1} \cup\{\infty\}$ be continuous and satisfy

(H1): $f(\cdot)$ is bounded from below and $\frac{f(B)}{\|B\|} \rightarrow \infty$, as $\|B\| \rightarrow \infty$.

Then, for any given $A \in R^{m n}$ and integer $k \geq 1$, there exist $\delta_{k}(A) \in \Delta_{k}$ such that

$$
f_{k}\left(A, \delta_{k}(A)\right)=\inf _{\delta_{k} \in \Delta_{k}} f_{k}\left(A, \delta_{k}\right) .
$$

Proof. By definition 2.1, we only need to show that the theorem holds for $k=1$ and $R_{1} f(\cdot)$ is continuous and satisfies the hypothesis $(\mathrm{H} 1)$, which are proved by the following lemmas.

Lemma 2.2. Let $f: R^{m n} \rightarrow R^{1} \cup\{\infty\}$ be continuous and satisfy the hypothesis (H1), then there exist $\delta_{1}(A) \in \Delta_{1}$ such that

$$
f_{1}\left(A, \delta_{1}(A)\right)=\inf _{\delta_{1} \in \Delta_{1}} f_{1}\left(A, \delta_{1}\right) .
$$

Proof. Without loss of generality, we may assume $R_{1} f(A)<f(A)$, since otherwise any $\hat{\delta}_{1}=(\theta, \phi, \tau, \gamma) \in S^{n-1} \times S^{m-1} \times S^{1} \times\{0\}$ is a solution of $(2.22)$.

Let $\left\{\delta^{i}=\left(\theta^{i}, \phi^{i}, \tau^{i}, \gamma^{i}\right)\right\}_{i=1}^{\infty}$ be a minimizing sequence of $f_{1}(A, \cdot)$ in $\Delta_{1}$. If $\left\{\gamma^{i}\right\}$ is bounded, then by extracting a convergent subsequence of $\left\{\delta^{i}\right\}$ and by the continuity of $f(\cdot)$, it is easily seen that the infimum in (2.22) is attainable.

We claim that $\left\{\gamma^{i}\right\}$ can not be unbounded. Otherwise, suppose $\gamma^{i} \rightarrow \infty$ as $i \rightarrow \infty$. Then, if $\sin ^{2}\left(\tau^{i}\right)$ and $\cos ^{2}\left(\tau^{i}\right)$ are bounded away from 0 , we have

$$
\begin{gathered}
\left\|A_{0}^{i}\right\|=\left\|A+\gamma^{i} \sin ^{2}\left(\tau^{i}\right) \phi^{i} \otimes \theta^{i}\right\| \rightarrow \infty, \\
\left\|A_{1}^{i}\right\|=\left\|A-\gamma^{i} \cos ^{2}\left(\tau^{i}\right) \phi^{i} \otimes \theta^{i}\right\| \rightarrow \infty,
\end{gathered}
$$

and thus, by $(\mathrm{H} 1), f_{1}\left(A, \delta^{i}\right) \rightarrow \infty$, which is a contradiction. On the other hand, suppose $\sin ^{2}\left(\tau^{i}\right) \rightarrow 0$ and $\sin ^{2}\left(\tau^{i}\right) \gamma^{i} \rightarrow b$ for some constant $b \in R^{1} \cup\{ \pm \infty\}$. If $b=0$, we have

$$
f_{1}\left(A, \delta^{i}(A)\right) \geq \cos ^{2}\left(\tau^{i}\right) f\left(A+\gamma^{i} \sin ^{2}\left(\tau^{i}\right) \phi^{i} \otimes \theta^{i}\right) \rightarrow f(A),
$$


this contradicts the assumption that $R_{1} f(A)<f(A)$. If $b \in R^{1} \backslash\{0\}$, since $\delta^{i}$ is a minimizing sequence and $f(\cdot)$ is bounded from below, we have

$$
\sin ^{2}\left(\tau^{i}\right) f\left(A-\gamma^{i} \cos ^{2}\left(\tau^{i}\right) \phi^{i} \otimes \theta^{i}\right) \leq C, \quad \forall i,
$$

where $C>0$ is a constant independent of $i$, thus, recalling that $\cos ^{2}\left(\tau^{i}\right) \rightarrow 1$ and $\gamma^{i} \rightarrow \infty$, as $i \rightarrow \infty$, we have, for sufficiently large $i$

$$
\begin{aligned}
f\left(A-\gamma^{i} \cos ^{2}\left(\tau^{i}\right) \phi^{i} \otimes \theta^{i}\right) \leq \frac{C}{|b|}\left|\gamma^{i}\right| & \leq \frac{2 C}{|b|}\left|\gamma^{i}\right| \cos ^{2}\left(\tau^{i}\right) \\
& \leq \frac{4 C}{|b|}\left\|A-\gamma^{i} \cos ^{2}\left(\tau^{i}\right) \phi^{i} \otimes \theta^{i}\right\| .
\end{aligned}
$$

This leads to a contradiction to (H1). $b= \pm \infty$ leads to (2.23) and (2.24), which by $(\mathrm{H} 1)$ contradict to the assumption that $\delta^{i}$ is a minimizing sequence. Similarly, the case $\cos ^{2}\left(\tau^{i}\right) \rightarrow 0$ can be ruled out.

Lemma 2.3. If $f(\cdot)$ is continuous and satisfies (H1), then its first order rank-one convex envelope $R_{1} f(\cdot)$ is also continuous and satisfies (H1).

Proof. Let $A$ be given, and let $\left\{B_{i}\right\}$ be a sequence satisfying $\lim _{i \rightarrow \infty}\left\|B_{i}\right\|=0$. By lemma 2.2, there exist $\delta \in \Delta_{1}$ and $\left\{\delta^{i}\right\} \subset \Delta_{1}$ such that

$$
\begin{aligned}
& R_{1} f(A)=\cos ^{2}(\tau) f\left(A+\gamma \sin ^{2}(\tau) \phi \otimes \theta\right)+ \\
& \sin ^{2}(\tau) f\left(A-\gamma \cos ^{2}(\tau) \phi \otimes \theta\right),
\end{aligned}
$$

and

$$
\begin{aligned}
R_{1} f\left(A+B_{i}\right)=\cos ^{2}\left(\tau^{i}\right) f\left(A+B_{i}+\gamma^{i} \sin ^{2}\left(\tau^{i}\right) \phi^{i} \otimes \theta^{i}\right)+ \\
\sin ^{2}\left(\tau^{i}\right) f\left(A+B_{i}-\gamma^{i} \cos ^{2}\left(\tau^{i}\right) \phi^{i} \otimes \theta^{i}\right) .
\end{aligned}
$$

Since we always have

$$
\begin{aligned}
R_{1} f\left(A+B_{i}\right) \leq \cos ^{2}(\tau) f\left(A+B_{i}+\right. & \left.\gamma \sin ^{2}(\tau) \phi \otimes \theta\right)+ \\
& \sin ^{2}(\tau) f\left(A+B_{i}-\gamma \cos ^{2}(\tau) \phi \otimes \theta\right),
\end{aligned}
$$

by $(2.28)$ and the continuity of $f(\cdot)$, we have

$$
\limsup _{i \rightarrow \infty} R_{1} f\left(A+B_{i}\right) \leq R_{1} f(A) .
$$


On the other hand, since $f\left(A+B_{i}\right)$ is bounded, with a similar argument as in the proof of lemma 2.2, it is easily verified that $\gamma^{i}$ must be bounded and thus there exists a subsequence of $\left\{\delta^{i}\right\}$, again denoted by $\left\{\delta^{i}\right\}$, and $\delta^{\infty} \in \Delta_{1}$ such that

$$
\lim _{i \rightarrow \infty} \delta^{i}=\delta^{\infty}, \quad \text { and } \quad \liminf _{i \rightarrow \infty} R_{1} f\left(A+B_{i}\right)=f_{1}\left(A, \delta^{\infty}\right) .
$$

By (2.20), this implies that

$$
\liminf _{i \rightarrow \infty} R_{1} f\left(A+B_{i}\right)=f_{1}\left(A, \delta^{\infty}\right) \geq R_{1} f(A) .
$$

The continuity of $R_{1} f(\cdot)$ follows from (2.31) and (2.32).

Now, let $\left\{A^{i}\right\}$ satisfy $\lim _{i \rightarrow \infty}\left\|A^{i}\right\|=\infty$ and $\delta^{i} \in \Delta_{1}$ be the corresponding minimizers. Let

$$
A_{0}^{i}=A^{i}+\gamma^{i} \sin ^{2}\left(\tau^{i}\right) \phi^{i} \otimes \theta^{i}, \quad A_{1}^{i}=A^{i}-\gamma^{i} \cos ^{2}\left(\tau^{i}\right) \phi^{i} \otimes \theta^{i} .
$$

We have

$$
\cos ^{2}\left(\tau^{i}\right)\left\|A_{0}^{i}\right\|+\sin ^{2}\left(\tau^{i}\right)\left\|A_{1}^{i}\right\| \geq\left\|A^{i}\right\| \rightarrow \infty
$$

and

$$
\frac{R_{1} f\left(A^{i}\right)}{\left\|A^{i}\right\|}=\frac{\cos ^{2}\left(\tau^{i}\right) f\left(A_{0}^{i}\right)+\sin ^{2}\left(\tau^{i}\right) f\left(A_{1}^{i}\right)}{\left\|\cos ^{2}\left(\tau^{i}\right) A_{0}^{i}+\sin ^{2}\left(\tau^{i}\right) A_{1}^{i}\right\|} .
$$

If both $\left\|A_{0}^{i}\right\|$ and $\left\|A_{1}^{i}\right\|$ are unbounded, then (2.35) and $f(\cdot)$ satisfies (H1) imply that

$$
\frac{R_{1} f\left(A^{i}\right)}{\left\|A^{i}\right\|} \rightarrow \infty
$$

If $\left\|A_{0}^{i}\right\|$ (or $\left.\left\|A_{1}^{i}\right\|\right)$ is bounded, then

$$
\frac{\left\|\sin ^{2}\left(\tau^{i}\right) A_{1}^{i}\right\|}{\left\|A^{i}\right\|} \rightarrow 1 \quad\left(\text { or } \quad \frac{\left\|\cos ^{2}\left(\tau^{i}\right) A_{0}^{i}\right\|}{\left\|A^{i}\right\|} \rightarrow 1\right)
$$

holds, this also leads to (2.36).

$R_{1} f(\cdot)$ is bounded from below is the direct consequence of the definition of $R_{1} f(\cdot)$ and $f(\cdot)$ is bounded from below.

Definition 2.4. Let $f \in C^{2}\left(R^{m n}\right) . \delta_{k}(A) \in \Delta_{k}$ is called a regular local minimizer of $f_{k}(A, \cdot)$ if

$$
\frac{\partial f_{k}\left(A, \delta_{k}(A)\right)}{\partial \delta_{k}}=0
$$


and

$$
\frac{\partial^{2} f_{k}\left(A, \delta_{k}(A)\right)}{\partial \delta_{k}^{2}}(\xi, \xi)>0, \quad \forall \xi \neq 0
$$

Lemma 2.4. Let $f \in C^{2}\left(R^{m n}\right)$. Suppose $\delta_{k}(A)$ is a regular local minimizer of $f_{k}(A, \cdot)$ for a given $A \in R^{m n}$. Then, there exists a neighborhood $\mathfrak{N}(A)$ of $A$ and a function $\delta_{k} \in C^{1}\left(\mathfrak{N}(A) ; \Delta_{k}\right)$ such that, for all $B \in \mathfrak{N}(A), \delta_{k}(B)$ is a regular local minimizer of $f_{k}(B, \cdot)$ and we have

$$
\frac{d f_{k}\left(A, \delta_{k}(A)\right)}{d A}=\left.\frac{\partial f_{k}\left(A, \delta_{k}\right)}{\partial A}\right|_{\delta_{k}=\delta_{k}(A)} .
$$

Proof. The existence of a unique continuously differentiable regular local minimizer $\delta_{k}(B)$ follows directly from the implicit function theorem [14], the definition of $f_{k}(\cdot)$ and $f \in C^{2}\left(R^{m n}\right)$. (2.40) is a consequence of (2.38).

Theorem 2.4. Let $f \in C^{2}\left(R^{m n}\right)$ and $A \in R^{m n}$. Suppose $\delta_{k}(B)$ is a regular local minimizer of $f_{k}(B, \cdot)$ and $R_{k} f(B)=f_{k}\left(B, \delta_{k}(B)\right)$ in a neighborhood of $A$. Then, we have

$$
\frac{d R_{k} f(A)}{d A}=\left.\frac{\partial f_{k}\left(A, \delta_{k}\right)}{\partial A}\right|_{\delta_{k}=\delta_{k}(A)}
$$

Proof. The theorem is a corollary of lemma 2.4 .

Remark 2.1. In applications, instead of the absolute minimizers of $f_{k}(A, \cdot)$ which define $R_{k} f(A)$, the local minimizers of $f_{k}(A, \cdot)$ can be used to describe metastable microstructures of laminates in laminates of order $k$.

\section{Multiscale computational model and Algorithm}

The stress free large deformations of martensite crystals, for example large deformations of shape memory alloys below the transformation temperature, are usually formed of microstructures which are locally laminates in laminates of finite order $k_{m}$, and in such a case the macroscopic deformation can be resolved by the solution of the relaxed problem

$$
\left(R_{k} P\right)\left\{\begin{array}{l}
\text { Find } u \in \mathbb{U}\left(u_{0} ; \Omega\right) \text { such that } \\
R_{k} F(u ; \Omega)=\inf _{v \in \mathbb{U}\left(u_{0} ; \Omega\right)} R_{k} F(v ; \Omega)
\end{array}\right.
$$


for $k \geq k_{m}$, and the microscopic information on the local laminates in laminates can be recovered by $\delta_{k}(\nabla u) \in \Delta_{k}$. Since the macroscopic deformation $u$ does not have fine oscillations, the numerical approximation of the relaxed problem is much easier than that of the original one.

To solve the $k$-th order rank-one convex relaxation problem $\left(R_{k} P\right)$ numerically, we use the finite element method to approximate the macroscopic deformation $u$. Let $\mathfrak{T}_{h}(\Omega)$ be a family of regular triangulations of $\Omega$ with mesh size $h$ [3]. For simplicity, we assume that for all $h$

$$
\bar{\Omega}_{h} \equiv \bigcup_{K \in \mathfrak{T}_{h}(\Omega)} K=\bar{\Omega} \quad \text { and } \quad \partial \Omega_{0 h}=\partial \Omega_{0},
$$

where $\partial \Omega_{0 h}$ is the union of all $n-1$ faces in $\mathfrak{T}_{h}(\Omega)$ whose interior has a nonempty intersection with $\partial \Omega_{0}$. Let

$$
\begin{aligned}
& \mathbb{U}_{h}\left(u_{0} ; \Omega\right)=\left\{u \in(C(\bar{\Omega}))^{m}:\left.u\right|_{K} \text { is affine } \forall K \in \mathfrak{T}_{h}(\Omega),\right. \\
& \left.\quad \text { and } u(x)=u_{0}(x), \text { if } x \text { is a node on } \partial \Omega_{0 h}\right\} .
\end{aligned}
$$

Then, the solution of $\left(R_{k} P\right)$ can be approximated by the solution of the following finite element discrete problem:

$$
\left(F R_{k} P\right)\left\{\begin{array}{l}
\text { Find } u_{h} \in \mathbb{U}_{h}\left(u_{0} ; \Omega\right) \text { such that } \\
R_{k} F\left(u_{h} ; \Omega\right)=\inf _{v_{h} \in \mathbb{U}_{h}\left(u_{0} ; \Omega\right)} R_{k} F\left(v_{h} ; \Omega\right) .
\end{array}\right.
$$

To reduce the mesh dependence of the finite element approximations, the mesh transformation method [20] can be applied. Let

$$
\begin{array}{r}
T(\Omega)=\left\{\text { bijections } g: \bar{\Omega} \rightarrow \bar{\Omega} \mid g \in\left(W^{1, \infty}(\Omega)\right)^{n}, g^{-1} \in\left(W^{1, \infty}(\Omega)\right)^{n}\right. \\
\left.g\left(\partial \Omega_{0}\right)=\partial \Omega_{0}, \text { and } \operatorname{det} \nabla g>0, \text { a.e. in } \Omega\right\}
\end{array}
$$

and

$$
T_{h}(\Omega)=\left\{g \in T(\Omega):\left.g\right|_{K} \text { is affine } \forall K \in \mathfrak{T}_{h}(\Omega)\right\} .
$$

Define the functional $R_{k} F(\cdot, \cdot ; \Omega)$ by

$$
\left.R_{k} F(\bar{u}, g ; \Omega)=\int_{\Omega} R_{k} f\left(\nabla \bar{u}(\bar{x})(\nabla g(\bar{x}))^{-1}\right)\right) \operatorname{det} \nabla g(\bar{x}) d \bar{x} .
$$

By changing the variables

$$
x=g(\bar{x}), \quad u(x)=\bar{u}\left(g^{-1}(x)\right),
$$


we are lead to the following discrete problem

$$
\left(M R_{k} P\right)\left\{\begin{array}{l}
\text { find }\left(\bar{u}_{h}, g_{h}\right) \in \mathbb{U}_{h}\left(u_{0} \circ g_{h} ; \Omega\right) \times T_{h}(\Omega) \text { such that } \\
R_{k} F\left(\bar{u}_{h}, g_{h} ; \Omega\right)=\inf _{(\bar{u}, g) \in \mathbb{U}_{h}\left(u_{0} \circ g ; \Omega\right) \times T_{h}(\Omega)} R_{k} F(\bar{u}, g ; \Omega) .
\end{array}\right.
$$

The discrete problem $\left(M R_{k} P\right)$ differs from that of $\left(F R_{k} P\right)$ in that, instead of minimizing the energy in a finite element function space defined on a fixed finite element mesh given by $\mathfrak{T}_{h}(\Omega)$, the energy is minimized among finite element functions defined on all admissible mesh distributions introduced by the mesh transformation mappings $g \in T_{h}(\Omega)$.

Remark 3.1. $T(\Omega)$ and $T_{h}(\Omega)$ can be modified to suit various kinds of boundary conditions.

Recalling that the $k$-th order rank-one convex envelope $R_{k} f$ is evaluated by (2.20) which works on the microscale, we see that the discrete problem $\left(M R_{k} P\right)$ is in fact a multiscale computational model. To compute the multiscale solution, the conjugate gradient method can be applied to solve $\left(M R_{k} P\right)$, where the values of $\left.\left.R_{k} f\left(\nabla \bar{u}(\bar{x})(\nabla g(\bar{x}))^{-1}\right)\right|_{\bar{x} \in K}\right)$ along with the microstructure information $\left.\left.\delta_{k}\left(\nabla \bar{u}(\bar{x})(\nabla g(\bar{x}))^{-1}\right)\right|_{\bar{x} \in K}\right)$ are obtained by solving the problem (2.20), and the gradients $\left.\left.\frac{d}{d A} R_{k} f\left(\nabla \bar{u}(\bar{x})(\nabla g(\bar{x}))^{-1}\right)\right|_{\bar{x} \in K}\right)$ are obtained by using the relation (2.41). The solution process can be summarized as the following algorithm:

(1): initial the deformation and mesh mapping $\left(\bar{u}_{h}^{0}, g_{h}^{0}\right)$;

(2): set $\left.A_{K}=\nabla \bar{u}_{h}^{0}(\bar{x})\left(\nabla g_{h}^{0}(\bar{x})\right)^{-1}\right)\left.\right|_{\bar{x} \in K}, \forall K \in \mathfrak{T}_{h}(\Omega)$;

(3): solve (2.20) to get $R_{k} f\left(A_{K}\right)$ and $\delta_{k}\left(A_{K}\right)$ by golden section method;

(4): compute the relaxed energy $R_{k} F\left(\bar{u}_{h}^{0}, g_{h}^{0} ; \Omega\right)$ by (3.6);

(5): compute $D_{h} R_{k} F$, the gradient of the relaxed energy respect to $\left(\bar{u}_{h}^{0}, g_{h}^{0}\right)$ by using the relation (2.41), the definition of $A_{K}$ and the chain rule;

(6): if $\left\|D_{h} R_{k} F\right\|<$ tolerance, take $\left(\bar{u}_{h}^{0}, g_{h}^{0}\right)$ and $\delta_{k}\left(A_{K}\right), \forall K \in \mathfrak{T}_{h}(\Omega)$ as the numerical solution, and terminate the process;

(7): compute the conjugate gradient direction $D_{h}^{c} R_{K} F$ and set $\left(\bar{u}_{h}^{1, \alpha}, g_{h}^{1, \alpha}\right)=$ $\left(\bar{u}_{h}^{0}, g_{h}^{0}\right)-\alpha \cdot D_{h}^{c} R_{K} F$

(8): using incomplete linear search to find an approximate minimizer $\bar{\alpha}$ of $e(\alpha)=R_{k} F\left(\bar{u}_{h}^{1, \alpha}, g_{h}^{1, \alpha} ; \Omega\right)$, where $R_{k} f\left(A_{K}^{\alpha}\right)$ and $\delta_{k}\left(A_{K}^{\alpha}\right), \forall K \in \mathfrak{T}_{h}(\Omega)$, with 
$\left.A_{K}^{\alpha}=\nabla \bar{u}_{h}^{1, \alpha}(\bar{x})\left(\nabla g_{h}^{1, \alpha}(\bar{x})\right)^{-1}\right)\left.\right|_{\bar{x} \in K}$, are obtained by solving $(2.20)$ using the golden section search;

(9): set $\left(\bar{u}_{h}^{0}, g_{h}^{0}\right)=\left(\bar{u}_{h}^{1, \bar{\alpha}}, g_{h}^{1, \bar{\alpha}}\right), R_{K} F\left(\bar{u}_{h}^{0}, g_{h}^{0} ; \Omega\right)=R_{K} F\left(\bar{u}_{h}^{1, \bar{\alpha}}, g_{h}^{1, \bar{\alpha}} ; \Omega\right), A_{K}=$ $A_{K}^{\bar{\alpha}}, \delta_{k}\left(A_{K}\right)=\delta_{k}\left(A_{K}^{\bar{\alpha}}\right), \forall K \in \mathfrak{T}_{h}(\Omega)$, go to step (5).

Numerical experiments on crystalline microstructure problems show that the elastic energy can often be completely relaxed even with fixed $h$ and $k$. That means there are abundant solutions to the finite order rank-one convex relaxed problem $\left(R_{k} P\right)$. Of course, in general, the algorithm can also be coupled with a mesh refinement and adaptivity strategy in applications to get a solution with smoother or particularly shaped macroscopic deformation.

\section{NUMERICAL EXPERIMENTS AND RESUlts}

Let $\Omega=D=\left(-X_{l} / 2, X_{l} / 2\right) \times\left(-Y_{l} / 2, Y_{l} / 2\right)$. Let $\mathfrak{T}_{N, M}(\Omega)=\mathfrak{T}_{h}(\Omega)=$ $\mathfrak{T}_{h}(D)$ be a family of regular triangulations introduced by the lines

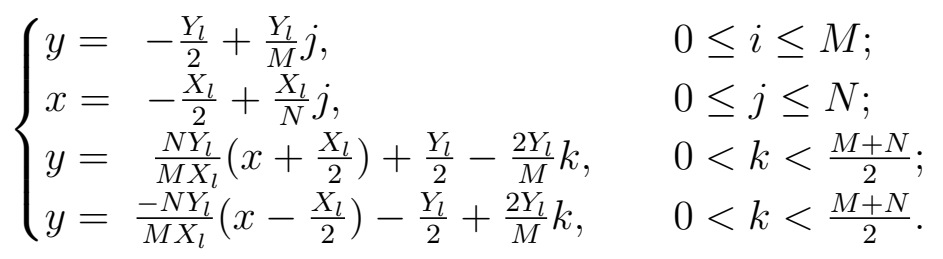

Consider a two dimensional model for elastic crystals with the energy density

$$
f(A)= \begin{cases}\Phi\left(A^{T} A\right), & \text { if } \operatorname{det} A>0 \\ +\infty, & \text { if } \operatorname{det} A \leq 0\end{cases}
$$

and

$$
\Phi(C)=\kappa_{1}(\operatorname{tr} C-2)^{2}+\kappa_{2} C_{12}^{2}+\kappa_{3}\left(\left(\frac{C_{11}-C_{22}}{2}\right)^{2}-\varepsilon^{2}\right)^{2}+\kappa_{4}\left(\log \frac{\operatorname{det} C}{1-\varepsilon^{2}}\right)^{2},
$$

where $C=\nabla u^{T} \nabla u$ is the right Cauchy-Green strain tensor, $\kappa_{i}>0, i=1,2,3,4$ are constant elastic moduli, and $\varepsilon>0$ is the transformation strain. Notice that here the energy density differs from the Ericksen-James energy density $[6,7,13]$ in that the energy density is infinity when $\operatorname{det} A \leq 0$, which is actually a physical requirement, and there is an additional term $\kappa_{4}\left(\log \frac{\operatorname{det} C}{1-\varepsilon^{2}}\right)^{2}$ to ensure that the energy density so defined is continuous in the whole space. The energy wells of the energy density defined by (4.2) and (4.3) are $S O(2) U_{0}$ and $S O(2) U_{1}$, while 
the energy wells of the Ericksen-James energy density are $O(2) U_{0}$ and $O(2) U_{1}$, where $O(2)=\left\{A \in R^{2 \times 2}: A^{T} A=I\right\}, S O(2)=\{A \in O(2): \operatorname{det} A=1\}$ and

$$
U_{0}=\left(\begin{array}{cc}
\sqrt{1-\varepsilon} & 0 \\
0 & \sqrt{1+\varepsilon}
\end{array}\right), \quad U_{1}=\left(\begin{array}{cc}
\sqrt{1+\varepsilon} & 0 \\
0 & \sqrt{1-\varepsilon}
\end{array}\right) .
$$

Let $\eta_{1}=\sqrt{1-\varepsilon}$ and $\eta_{2}=\sqrt{1+\varepsilon}$ and let

$$
R^{ \pm}=\left(\begin{array}{cc}
\eta_{1} \eta_{2} & \pm \varepsilon \\
\mp \varepsilon & \eta_{1} \eta_{2}
\end{array}\right)
$$

then it is easily verified that

$$
R^{ \pm} U_{1}=U_{0}+\mathbf{a}^{ \pm} \otimes \mathbf{n}^{ \pm}
$$

where $\mathbf{a}^{ \pm}=\sqrt{2} \varepsilon\left(\eta_{1}, \mp \eta_{2}\right)^{T}$ and $\mathbf{n}^{ \pm}=\frac{1}{\sqrt{2}}(1, \pm 1)^{T}$, that is $U_{0}$ and $R^{ \pm} U_{1}$ are in rank-one connection.

In our numerical experiments, we set $\kappa_{1}=10, \kappa_{2}=3, \kappa_{3}=1, \kappa_{4}=10^{-8}$ and $\varepsilon=0.1$.

Theorem 4.1. Let $A \in R^{n \times n}$ and $B=A+\mathbf{b} \otimes \mathbf{m}$ for some $\mathbf{b}, \mathbf{m} \in R^{n}$. Suppose $\operatorname{det} A>0$ and $\operatorname{det} B>0$. Then, $\operatorname{det}((1-\lambda) A+\lambda B)>0$ for all $\lambda \in[0,1]$.

Proof. By the fact that the map $R^{n \times n} \ni A \rightarrow \operatorname{det} A$ is rank-one affine and $\operatorname{rank}(A-B) \leq 1$, we have

$$
\operatorname{det}((1-\lambda) A+\lambda B)=(1-\lambda) \operatorname{det} A+\lambda \operatorname{det} B .
$$

Equation (4.7) implies that $\operatorname{det}((1-\lambda) A+\lambda B) \geq \min \{\operatorname{det} A, \operatorname{det} B\}>0$, thus the theorem follows.

By Theorem 4.1, for the energy density satisfying $f(A)=+\infty$ if $\operatorname{det} A \leq 0$, the finite order rank-one convex envelopes can be equivalently defined as follows.

Definition 4.1. The sequence

$$
\left(\lambda_{i_{1}}, \lambda_{i_{1} i_{2}}, \ldots, \lambda_{i_{1} i_{2} \cdots i_{k}}, A_{i_{1} i_{2} \cdots i_{k}}\right), \quad i_{\nu} \in\{0,1\}, \quad 1 \leq \nu \leq k
$$

is said to satisfy $\left(\mathrm{R}_{k}^{+}\right)$if it satisfies $\left(\mathrm{R}_{k}\right)$ and a further condition

(iii): $\operatorname{det} A_{i_{1} \cdots i_{k}}>0, \quad \forall i_{\nu} \in\{0,1\}, \quad 1 \leq \nu \leq k$. 
Definition 4.2. $R_{k} f: R^{m n} \rightarrow R^{1} \cup\{\infty\}$ is said to be the $k$-th order rank-one convex envelope of $f$, if

$$
R_{k} f(A)=\left\{\begin{array}{cl}
\inf \left\{\sum_{i_{1}, \ldots, i_{k}=0}^{1} \lambda_{i_{1}} \lambda_{i_{1} i_{2}} \cdots \lambda_{i_{1} i_{2} \cdots i_{k}} f\left(A_{i_{1} i_{2} \cdots i_{k}}\right):\right. & \\
\sum_{i_{1}, \ldots, i_{k}=0}^{1} \lambda_{i_{1}} \lambda_{i_{1} i_{2}} \cdots \lambda_{i_{1} i_{2} \cdots i_{k}} A_{i_{1} i_{2} \cdots i_{k}}=A, & \\
\left.\left(\lambda_{i_{1}}, \lambda_{i_{1} i_{2}}, \ldots, \lambda_{i_{1} i_{2} \cdots i_{k}}, A_{i_{1} i_{2} \cdots i_{k}}\right) \text { satisfy }\left(\mathrm{R}_{k}^{+}\right)\right\}, & \text {if } \operatorname{det} A>0 \\
+\infty, & \text { if } \operatorname{det} A \leq 0
\end{array}\right.
$$

It follows from Theorem 4.1 and Definition 4.2 that the microstructures consists of laminates in laminates defined by the finite order rank-one convex envelopes are physically admissible, i.e. the determinants of the deformation gradients of the laminates on various levels are all positive.

In our numerical experiments, the first order rank-one convex envelope $R_{1} f(\cdot)$ defined by Definition 4.2 is used. The algorithm given in Section 3 is applied to compute the 2-scale numerical solution, which consists of piece-wise simple laminated microstructures with the average deformation gradients of the form

$$
R(\alpha) A_{\lambda}^{-}=R(\alpha)\left((1-\lambda) U_{0}+\lambda R^{-} U_{1}\right),
$$

or

$$
R(\beta) A_{\mu}^{+}=R(\beta)\left((1-\mu) U_{0}+\mu R^{+} U_{1}\right),
$$

where $R(\theta) \in S O(2)$ is the rotation matrix with rotation angle $\theta$. It is easily verified that $\operatorname{rank}\left(R\left(\alpha_{\lambda, \mu}\right) A_{\lambda}^{-}-A_{\mu}^{+}\right)=1$, where

$$
\alpha_{\lambda, \mu}=\arctan \left(\frac{a \sqrt{a^{2}+b^{2}-\eta_{1}^{2} \eta_{2}^{2}}-b \eta_{1} \eta_{2}}{b \sqrt{a^{2}+b^{2}-\eta_{1}^{2} \eta_{2}^{2}}+a \eta_{1} \eta_{2}}\right),
$$

and where in (4.11) $a=\eta_{1} \eta_{2}\left(1-2 \lambda \mu \varepsilon^{2}\right), b=(\lambda+\mu) \varepsilon-2 \lambda \mu \varepsilon^{3}$.

Example 1. Take $X_{l}=32, Y_{l}=2, M=1$ and $N=16$. Let the initial mesh transformation map be the identity map, that is $g_{0 h}(\bar{x})=\bar{x}$. Let

$$
r(\tau, \theta, \bar{x})=\tau X_{l} \theta^{-1}-\bar{x}_{2}, \quad \theta(\bar{x})=\left(\frac{\bar{x}_{1}}{X_{l}}+\frac{1}{2}\right) \theta
$$

and let

$$
\bar{u}_{0}(\bar{x})=\left(-0.5 X_{l}+r\left(\tau, \theta, \bar{x}_{2}\right) \sin \theta(\bar{x}),-0.5 Y_{l}+r\left(\tau, \theta, \bar{x}_{2}\right)(1-\cos \theta(\bar{x}))^{T},\right.
$$


which maps $D$ into a part of an upward bending annular region with the central angle $\theta$. Take the interpolation function $\bar{u}_{0 h}$ of $\bar{u}_{0}$ in $\mathfrak{T}_{h}(D)$ as the initial deformation. The boundary conditions for $g_{h}$ and $\bar{u}_{h}$ are given by

$$
\begin{aligned}
g_{h}\left( \pm 0.5 X_{l}, \pm 0.5 Y_{l}\right) & =\left( \pm 0.5 X_{l}, \pm 0.5 Y_{l}\right)^{T} \\
\bar{u}_{h}\left(-0.5 X_{l},-0.5 Y_{l}\right) & =\left(-0.5 X_{l},-0.5 Y_{l}\right)^{T} \\
\left(\bar{u}_{h}\left(-0.5 X_{l}, 0.5 Y_{l}\right)\right)_{1} & =-0.5 X_{l} .
\end{aligned}
$$

The numerical experiments show that, for any given $\theta \in[0, \pi / 3]$ and $\tau \in$ $(1-\varepsilon, 1)$, the algorithm given in Section 3 leads to a stress free large deformation consisting of piecewise simple laminated microstructures. Similar numerical results can be obtained, if we take

$$
\bar{u}_{0}(\bar{x})=\left(-0.5 X_{l}+r\left(\tau, \theta, \bar{x}_{2}\right) \sin \theta(\bar{x}), 0.5 Y_{l}-r\left(\tau, \theta, \bar{x}_{2}\right)(1-\cos \theta(\bar{x}))^{T},\right.
$$

whose image is a part of a downward bending annular region with the central angle $\theta$.

In Figure 1, the macroscopic deformed configurations of the numerical results for some given $\theta$ are shown, where the gradients $A_{K}$ of the macroscopic deformation on the black and white elements are approximately of the form

$$
R\left(\alpha_{K}\right) A_{\lambda_{K}}^{-} \text {and } R\left(\beta_{K}\right) A_{\mu_{K}}^{+}, \quad \forall K \in \mathfrak{T}_{h}(D)
$$

respectively. Furthermore, let $K$ be ordered from left to right, then the following relations hold approximately as shown in Table 1

$$
\begin{array}{lll}
\mu_{K}=\lambda_{K+1}, & \text { if } K=0 \quad \bmod (4) ; \\
\mu_{K}=\lambda_{K-1}, & \text { if } K=3 \quad \bmod (4) ; \\
\alpha_{K}=\alpha_{K+1}, & \text { for } K=1,3 \bmod (4) ; \\
\alpha_{K}=\alpha_{K-4}+\Delta \alpha_{j}, & \text { if } K=4 j+i, \text { for } i \in\{1,2,3,4\} .
\end{array}
$$

This agrees with the theory. In fact, by (4.11), in a 4 elements block $K \in$ $\{4 j, 4 j+1,4 j+2,4 j+3\}$ the rotation angle increment $\Delta \alpha_{j}$ is given by

$$
\Delta \alpha_{j}=\alpha_{\mu_{4 j}, \lambda_{4 j+1}}-\alpha_{\lambda_{4 j+2}, \mu_{4 j+3}}=\alpha_{\mu_{4 j}, \mu_{4 j}}-\alpha_{\mu_{4 j+3}, \mu_{4 j+3}} .
$$

Obviously, macroscopic deformation with varying curvature can be obtained if $\mu_{4 j}$ and $\mu_{4 j+3}$ varies from block to block accordingly. 

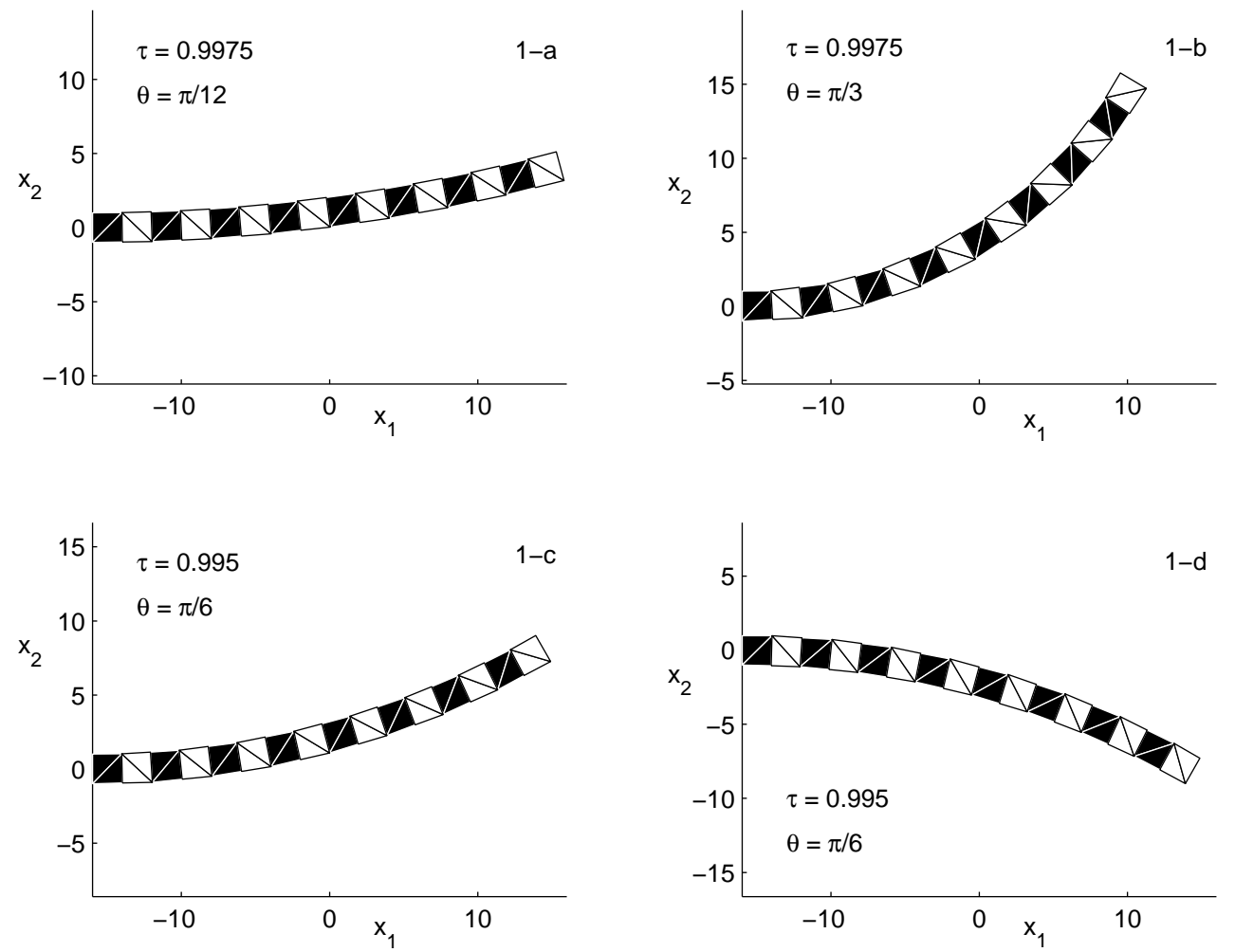

Figure 1. The macroscopic deformed configurations for various $\theta$.

TABLE 1. Numerical results corresponding to Figure 1 (a)-(d).

\begin{tabular}{|c|c|c|c|c|}
\hline & $(\mathrm{a}) \theta=\pi / 12$ & $(\mathrm{~b}) \theta=\pi / 3$ & $(\mathrm{c}) \theta=\pi / 6$ & $(\mathrm{~d}) \theta=\pi / 6$ \\
\hline$\mu_{K}, K=0 \bmod (4)$ & $\approx 0.42$ & $\approx 0.17$ & $\approx 0.31$ & $\approx 0.64$ \\
\hline$\mu_{K}, K=3 \bmod (4)$ & $\approx 0.58$ & $\approx 0.83$ & $\approx 0.64$ & $\approx 0.31$ \\
\hline$\alpha_{1} \approx \alpha_{2} \approx$ & $-4.28 \times 10^{-2}$ & $-2.71 \times 10^{-2}$ & $-3.59 \times 10^{-2}$ & $-5.72 \times 10^{-2}$ \\
\hline$\alpha_{3} \approx \alpha_{4} \approx$ & $6.91 \times 10^{-2}$ & $1.37 \times 10^{-1}$ & $8.81 \times 10^{-2}$ & $-2.90 \times 10^{-4}$ \\
\hline$\Delta \alpha_{j} \approx$ & $3.33 \times 10^{-2}$ & $1.33 \times 10^{-1}$ & $6.64 \times 10^{-2}$ & $-6.66 \times 10^{-2}$ \\
\hline elastic energy & $1.14 \times 10^{-11}$ & $1.22 \times 10^{-11}$ & $5.48 \times 10^{-12}$ & $3.07 \times 10^{-12}$ \\
\hline$L^{2}$-norm of stress & $9.26 \times 10^{-7}$ & $7.74 \times 10^{-7}$ & $9.97 \times 10^{-7}$ & $8.14 \times 10^{-7}$ \\
\hline
\end{tabular}

Example 2. Let $X_{l}=36, Y_{l}=2, M=1$ and $N=18$, and let the initial mesh transformation map be $g_{0 h}(\bar{x})=\bar{x}$ as in Example 1. Let $\bar{u}_{0}\left(D^{+}\right)$and $\bar{u}_{0}\left(D^{-}\right)$be a upward and downward bending annular region with central angle 
$\theta$ respectively, where $D^{+}=\left\{x \in D: x_{1} \geq 2\right\}$ and $D^{-}=\left\{x \in D: x_{1} \leq-2\right\}$, and let $\left.\bar{u}_{0}(\bar{x})\right|_{D \backslash\left(D^{+} \cup D^{-}\right)}=\bar{x}$. Let $\bar{u}_{0 h}$ be the interpolation of $\bar{u}_{0}$ in $\mathfrak{T}_{18,1}(D)$. Let the boundary condition of $g_{0 h}$ be given by (4.12). Then, the algorithm given in Section 3 leads to a stress free large deformation, as shown in Figure 2 (a), which again consists of piecewise simple laminated microstructures of the form (4.15) with the relations (4.17)-(4.19) approximately satisfied. The numerical results on $\alpha_{K}, \beta_{K}, \lambda_{K}$ and $\mu_{K}$ for Example 2 are shown in Table 2.
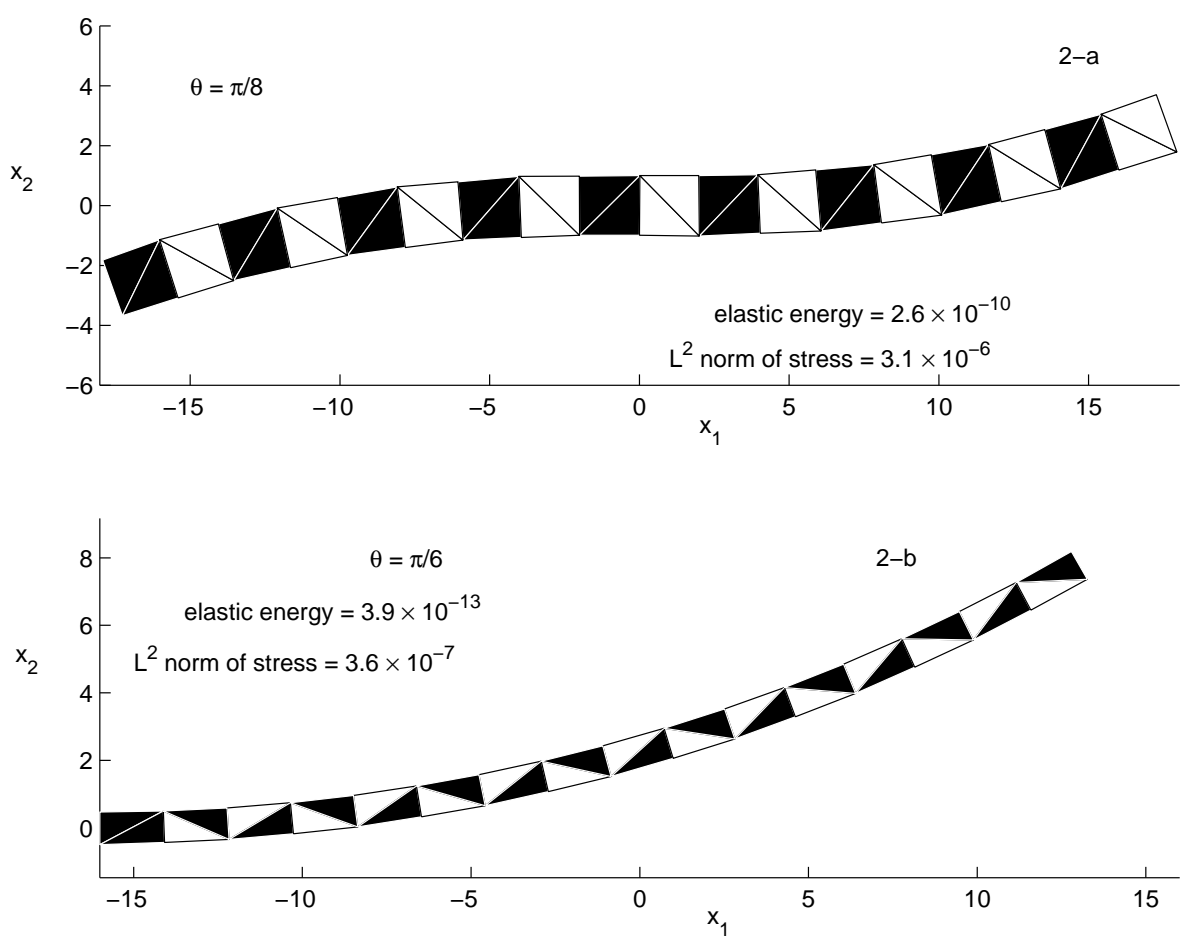

Figure 2. The macroscopic deformed configurations for Example 2 and 3 .

Example 1 and Example 2 show that, in general, the solution to the finite order rank-one convex relaxation problem (3.1) is non-unique. In fact, the above numerical experiments show that there are infinitely many piecewise simple laminated microstructures which can macroscopically deform a thin string made of martensite crystal into a stress free curve with its curvature bounded by a constant depending on the string width and the transformation strain $\varepsilon$.

We point out here that the condition $f(A)=+\infty$ if $\operatorname{det} A \leq 0$ is necessary to guarantee that the numerical solution is physically admissible. In fact, for 
TABLE 2. Numerical results corresponding to Figure 2 (a).

\begin{tabular}{|c|c|c|c|c|c|c|c|c|}
\hline \multirow{2}{*}{$\mathrm{i}$} & \multicolumn{2}{|c|}{$K=4 i+1$} & \multicolumn{2}{c|}{$K=4 i+2$} & \multicolumn{2}{c|}{$K=4 i+3$} & \multicolumn{2}{c|}{$K=4 i+4$} \\
\cline { 2 - 9 } & $\alpha_{K}$ & $\lambda_{K}$ & $\alpha_{K}$ & $\lambda_{K}$ & $\beta_{K}$ & $\mu_{K}$ & $\beta_{K}$ & $\mu_{K}$ \\
\hline 0 & 0.2809 & 0.6084 & 0.2808 & 0.2744 & 0.3317 & 0.2745 & 0.3318 & 0.7059 \\
\hline 1 & 0.1947 & 0.7059 & 0.1947 & 0.2516 & 0.2414 & 0.2516 & 0.2413 & 0.6894 \\
\hline 2 & 0.1076 & 0.6893 & 0.1076 & 0.2543 & 0.1548 & 0.2543 & 0.1548 & 0.7068 \\
\hline 3 & 0.01745 & 0.7067 & 0.01741 & 0.2613 & 0.06600 & 0.2613 & 0.06598 & 0.6297 \\
\hline 4 & -0.05542 & 0.6297 & -0.05544 & 0.5144 & 0.04257 & 0.5144 & 0.04257 & 0.3744 \\
\hline 5 & -0.02775 & 0.3744 & -0.02772 & 0.6991 & 0.1080 & 0.6991 & 0.1080 & 0.2355 \\
\hline 6 & 0.06437 & 0.2355 & 0.06440 & 0.6923 & 0.1988 & 0.6923 & 0.1983 & 0.2611 \\
\hline 7 & 0.1502 & 0.2611 & 0.1502 & 0.6954 & 0.2853 & 0.6954 & 0.2853 & 0.2396 \\
\hline 8 & 0.2408 & 0.2396 & 0.2408 & 0.6843 & 0.3735 & 0.6843 & 0.3736 & 0.3272 \\
\hline
\end{tabular}

the Ericksen-James energy density, other than the physically admissible energy wells $U_{0}$ and $U_{1}$, there are two non-physical energy wells $Q U_{0}$ and $Q U_{1}$, where $Q \in O(2)$ is a diagonal matrix with entries 1 and -1 , and it is not difficult to verified that $\operatorname{rank}\left(U_{0} \pm Q U_{0}\right)=\operatorname{rank}\left(U_{1} \pm Q U_{1}\right)=1$, and thus, if the gradient of the macroscopic deformation has the form

$$
A_{\lambda, \alpha}^{ \pm, 0}=R(\alpha)\left((1-\lambda) U_{0} \pm \lambda Q U_{0}\right) \quad \text { or } \quad A_{\lambda, \alpha}^{ \pm, 1}=R(\alpha)\left((1-\lambda) U_{1} \pm \lambda Q U_{1}\right),
$$

then a non-physical simple laminated microstructure with deformation gradients

$$
R(\alpha) U_{0} \text { and } \mp R(\alpha) Q U_{0} \quad \text { or } \quad R(\alpha) U_{1} \text { and } \mp R(\alpha) Q U_{1}
$$

and volume fractions $(1-\lambda)$ and $\lambda$ is formed correspondingly. Noticing that if the volume fraction $\lambda<0.5$, that is if the contribution of $Q U_{i}$ part to the overall deformation is relatively smaller, then $\operatorname{det} A_{\lambda, \alpha}^{ \pm, i}>0$, we see that even physically admissible macroscopic deformation can consists of non-physical laminated microstructures.

Example 3. Consider the Ericksen-James energy density, that is $f(A)=\Phi\left(A^{T} A\right)$ with $\kappa_{4}=0$. Take $X_{l}=32, Y_{l}=1, M=1$ and $N=16$. Let $g_{0 h}(\bar{x})=\bar{x}$. Let $\bar{u}_{0}$ and $\bar{u}_{0 h}$ be defined in the same way as in Example 1 with $\tau=0.95$ and $\theta=\pi / 6$. Then, the algorithm leads to a non-physical solution shown in Figure 2 (b), where the gradients of the macroscopic deformation on the black and white elements 
are approximately of the form $A_{\lambda, \alpha}^{+, 0}$ and $A_{\lambda, \alpha}^{-, 1}$ respectively, with $\lambda \approx 0.055$ and $\alpha$ satisfying approximately the relation (4.19) for $\Delta \alpha_{j} \approx 0.065$. For example the gradient $A_{9}$ of the macroscopic deformation on the 9-th element is

$$
A_{9}=\left(\begin{array}{cc}
0.92387307 & -0.13035023 \\
0.13098280 & 0.93965785
\end{array}\right)
$$

and for $\alpha=0.1394, \lambda=0.05515$

$$
A_{\lambda, \alpha}^{-, 1}=\left(\begin{array}{cc}
0.92407349 & -0.13181856 \\
0.12965678 & 0.93948064
\end{array}\right) .
$$

\section{REFERENCES}

[1] J. M. Ball and R. D. James, Fine phase mixtures as minimizers of energy. Arch. Rat. Mech. Anal., 100(1987)1, 13-52.

[2] J. M. Ball and R. D. James, Proposed experimental test of a theory of fine microstructure and the two-well problem. Phil. Trans. R. Soc. London 338A(1992), 389-450.

[3] P.G. Ciarlet, The Finite Element Method for Elliptic Problems. North-Holland, Amsterdam, 1978.

[4] P. G. Ciarlet, Mathematical Elasticity, Volume 1: Three Dimensional Elasticity. NorthHolland, Amsterdam, 1988.

[5] C. Collins and M Luskin, The computation of the austenitic-martensitic phase transition. In Partial Differential Equations and Continuum Models of Phase Transitions Lecture Nodes in Physics, Vol. 344, M. Rascle, D. Serre and M. Slemrod, eds, SpringerVerlag, New York, (1989), pp. 34-50.

[6] C. Collins, Computation of twinning, in Microstructure and Phase Transitions, IMA Volumes in Mathematics and Its Applications, Vol.54, J. Ericksen, R. James, D. Kinderlehrer and M. Luskin, eds, Springer-Verlag, New York, (1993), pp. 39-50.

[7] C. Collins, M. Luskin and J. Riordan, Computational results for a two-dimensional model of crystalline microstructure, in Microstructure and Phase Transitions, IMA Volumes in Mathematics and Its Applications, Vol.54, J. Ericksen, R. James, D. Kinderlehrer and M. Luskin, eds, Springer-Verlag, New York, (1993), pp. 51-56.

[8] B. Dacorogna, Direct Methods in the Calculus of Variations, Applied Mathematical Sciences, 78, Springer-Verlag, Berlin, 1989.

[9] G. Dolzmann, Numerical computation of rank-one convex envelopes. SIAM J. Numer. Anal., 36(1999), 1621-1635. 
[10] G. Dolzmann and N. J. Walkington, Estimates for numerical approximations of rank-one convex envelopes. Numer. Math., 85 (2000), pp. 647-663.

[11] M.K. Gobbert and A. Prohl, A comparison of classical and new finite element methods for the computation of laminated microstructure, Appl. Numer. Math. 36(2001), pp.155178.

[12] M. K. Gobbert and A. Prohl, A discontinuous finite element method for solving a multi-well problem. SIAM J Numer. Anal. 37(1999), pp. 246-268.

[13] P.-A. Gremaud, Numerical analysis of a nonconvex variational problem related to solidsolid phase transitions. SIAM J. Numer. Anal., 31(1994), 111-127.

[14] S. Lang, Real and Functional Analysis. Springer-Verlag, New York, 1993.

[15] Z.-P. Li, Simultaneous numerical approximation of microstructures and relaxed minimizers. Numer. Math., 78(1997), pp.21-38.

[16] Z.-P. Li, Laminated microstructure in a variational problem with a non-rank-one connected double well potential. J. Math. Anal. Appl., 217(1998), pp.490-500.

[17] Z.-P. Li, Rotational transformation method and some numerical techniques for the computation of microstructures. Math. Models Meth. Appl. Sci., 8(1998), 985-1002.

[18] Z.-P. Li, Finite order rank-one convex envelopes and computation of microstructures with laminates in laminates. BIT Numer. Math., 40(4)(2000), pp.745-761.

[19] Z.-P. Li, A periodic relaxation method for computing microstructures. Appl. Numer. Math., 32(2000), 291-303.

[20] Z.-P. Li, A mesh transformation method for computing microstructures. Numer. Math., 89(2001), pp. 511-533.

[21] Z.-P. Li, A numerical study on the scale of laminated microstructure with surface energy. Materials Science and Engineering, A 343(2003), pp. 182-193.

[22] Z.-P. Li, Numerical justification of branched laminated microstructure with surface energy. SIAM J. Sci. Compt., 24(2003), pp. 1054-1075.

[23] M. Luskin, On the computation of crystalline microstructure. Acta Numerica, 5(1996), 191-257.

[24] C. B. Morrey, Multiple Integrals in the Calculus of Variations, Springer, New York, 1966.

[25] P. Pedregal, Numerical approximation of parameterized Measures, Numer. Funct. Anal. Optim., 16(1995), 1049-1066. 